\title{
Autoantibodies to PDGFR are profibrotic in vivo
}

The SSc
phenotype
was confirmed
by increased
collagen
deposition
and markers
of fibroblast
activation

Studies using a bioengineered skin-humanized mouse model of systemic sclerosis $(\mathrm{SSc})$ reveal that autoantibodies that bind and stimulate platelet-derived growth factor receptor (PDGFR) induce fibrosis in vivo.

Creation of the model entailed the ex vivo generation of bioengineered skin containing human keratinocytes and fibroblasts isolated from samples taken from patients with SSc or healthy donors, which was then engrafted onto the backs of severe combined immunodeficiency (SCID) mice. "The methodology is exciting because it allows the graft of a single human skin sample in several mice, thus permitting different in vivo experimental conditions on the same tissue," explains Armando Gabrielli, corresponding author of the paper reporting the findings.

Immunohistochemistry analysis confirmed that the skin grafts derived from patients with SSc retained a fibrotic disease phenotype up to 16 weeks after engraftment, but gradually lost these features and by 24 weeks were indistinguishable from skin grafts generated from healthy donor samples. This finding suggested that additional factors absent from the skin grafts were needed to maintain the SSc phenotype; thus, the researchers investigated the role of agonistic anti-PDGFR autoantibodies, on the basis of previous work demonstrating their presence in the serum of patients with SSc and other studies implicating PDGF-PDGFR signalling in fibrosis and SSc.

In animals carrying healthy donor skin grafts, "the injection of total IgG purified from serum of [SSc]patients induced fibrosis of the human skin graft, whereas IgG purified from healthy controls did not," reports Gabrielli. The SSc phenotype was confirmed by increased collagen deposition and markers of fibroblast activation. Notably, oral administration of the tyrosine kinase inhibitor nilotinib before the injection of SSc IgG ameliorated the disease phenotype. Further reinforcing the role of PDGFR activation in mediating the effects of SSc IgG, injection of agonistic human monoclonal antiPDGFR antibodies (generated from a single patient with SSc) was also

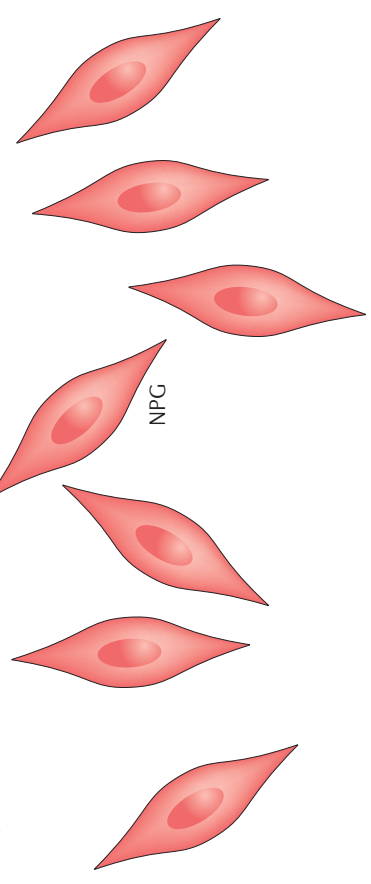
sufficient to induce fibrosis in the skin grafts in vivo.

"We plan to use this novel mouse model of skin fibrosis to test the antifibrotic activity of drugs in vivo and to generate further animal models of systemic sclerosis [using] our agonistic monoclonal anti-PDGFR antibodies," says Gabrielli.

\section{Sarah Onuora}

ORIGINAL ARTICLE Luchetti, M. M. et al. Induction of scleroderma fibrosis in skin-humanized mice by anti-Platelet-Derived Growth Factor receptor agonistic autoantibodies. Arthritis Rheumatol. http://dx.doi.org/10.1002/art.39728 (2016) 